\title{
Affective Expressions of Machines
}

\author{
Christoph Bartneck \\ IPO - Center for User System Interaction \\ Eindhoven University of Technology \\ Post Box 513, 5600 MB Einhoven, \\ The Netherlands \\ christoph@bartneck.de \\ Philips Research Laboratories \\ Prof. Holstlaan 4 \\ 5656 AA Eindhoven \\ The Netherlands
}

\begin{abstract}
Emotions should play an important role in the design of interfaces because people interact with machines as if they were social actors [4]. We developed and tested a model for the convincingness of affective expressions, based on Fogg and Hsiang Tseng [3]. The empirical data did not support our original model. Furthermore, the experiment investigated if the type of emotion (happiness, sadness, anger, surprise, fear and disgust), knowledge about the source (human or machine), the level of abstraction (natural face, computer rendered face and matrix face) and medium of presentation (visual, audio/visual, audio) of an affective expression influences its convincingness and distinctness. Only the type of emotion and multimedia presentations had an effect on convincingness. The distinctness of an expression depends on the abstraction and the media through which it is presented.
\end{abstract}

\section{Keywords}

Affective expressions, emotion, speech, face, music, convincingness, distinctness, abstraction, modality

\section{INTRODUCTION}

Philips Research Laboratories in co-operation with the IPO has set up the Affective Machines project to improving the user experience by enabling machines to use affective expressions. This paper describes the results of the first phase which focuses on the expressions itself. In the two upcoming phases an affective system architecture and a prototype will be build and evaluated. A more detailed description of this study is available elsewhere [1].

\section{THE EXPERIMENT}

The most important attribute of affective expressions of machines is their convincingness. We consider the concept of convincingness as an extension of Fogg's and Hsiang Tseng's concept of believability [3], that is based on trustworthiness and expertise. We added the intensity and the distinctness of the stimuli to their original definition, because they are particularly important for affective systems. Distinctness is the attribute of the expression, that is measured by the recognition accuracy of the subjects. The expertise of the system is measured by the perceived appropriateness of its expressions. In the experiment subjects observed a dice game and evaluated the affective expressions of one of the players. Software presented the expressions to ensure consistency. It also showed the game and the questions. To test whether the source of the expression influences its perception the player was either a human or a computer. The software showed a picture of either a human or a computer and clearly labeled them.

Machines need a clear vocabulary of emotions that provide enough complexity to act appropriate in most situations. We tested the basic 6 categories: happiness, sadness, anger, surprise, fear and disgust. The technical abilities of machines might differ (e.g. screen size and resolution). Therefore we tested 3 levels of abstractions of facial expressions (see Figure 1). Machines might also differ in the available media (e.g. screen or speaker). A combination of media might be perceived differently. Hence, we compared multimedia expression with their single medium expressions. We expected the context in which each expression occurred to have influence on its perception. Therefore the software paired each stimulus with its specific context. To reduce the complexity of the experiment we limited certain factors to certain conditions.

Convincingness, expertise, trustworthiness and intensity were measured by answering a question (e.g. "How convincing is this expression?") on a 1-7. The distinctness of an expression was measured by the recognition accuracy of the subjects (forced choice between the 7 categories).

We used the face of an actor, Baldi [2], an optimized matrix face and audio (abstract music) as stimuli:
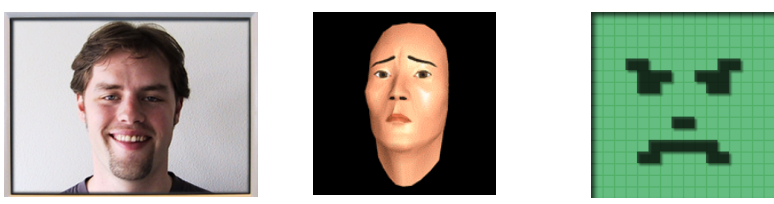

Figure 1: Examples of the 3 visual abstraction levels

33 subjects participated in the experiment. They were explicitly instructed to distinguish between trustworthiness 
and convincingness and between the type of player (human or computer).

\section{Results}

Table 1 presents the correlation matrix for variables predicting convincingness. $84.1 \%$ of the variance in convincingness can be predicted from distinctness, intensity, trustworthiness and expertise. Distinctness is only weakly correlated $(\mathrm{r}=.380)$ to convincingness and is not a significant (sig. $=.107)$ predictor. Both, convincingness $(\mathrm{r}=.874)$ and intensity $(\mathrm{r}=.736)$ are strongly correlated to trustworthiness. Intensity is not a significant (sig.=.462) predictor for convincingness when trustworthiness is already considered in the analyses (collinearity). Trustworthiness alone predicts $75.6 \%$ of the variance in convincingness.

\begin{tabular}{|l|r|r|r|r|}
\hline & \multicolumn{1}{|l|}{ Conv. } & \multicolumn{1}{l|}{ Distinct. } & \multicolumn{1}{l|}{ Intensity } & Expertertise \\
\hline Distinct. & $0.380^{*}$ & - & & \\
\hline Intens. & 0.677 & $0.280^{*}$ & - & \\
\hline Expert. & 0.787 & 0.377 & 0.418 & - \\
\hline Trustw. & 0.874 & $0.180^{*}$ & 0.736 & 0.666 \\
\hline
\end{tabular}

Table 1: Pearson correlation coefficients

The type of emotion has significant influence on convincingness. Surprise (5.68) and happiness (5.71) were more convincing than sadness (5.25), disgust (5.05) and anger (4.67) which were more convincing than fear (4.02).

Distinctness is significantly influenced by the type of emotion expressed. The scores for sadness (90\%) were above the ones for anger (71\%). There was no significant difference within the "higher" scores, sadness (90\%), happiness (95\%) and surprise (93\%) and within the "lower" scores, disgust (68\%) anger (71\%) and fear (70\%).

Knowledge about the source of the emotional expression has no significant influence on its convincingness. The abstraction of an emotional expression has no significant influence on its convincingness. Only the scores for distinctness were influenced significantly (Baldi 94\%, Natural 89\% and Matrix 77\%).

The medium used to express an emotion has significant influence on its convincingness. Visual (5.10) and audio/visual (5.19) expressions were slightly more convincing than audio expressions (4.77). Distinctness was not significantly influenced.

\section{Discussion}

Distinctness is, against our expectations, not a significant predictor for convincingness. It was impossible for the subjects to evaluate their choice, because we did not provide them with feedback about the correctness of their interpretation. Therefore, they rated the convincingness of the expressions independent of whether they interpreted the emotion correctly or not. They could make up their own interpretation of why this expression makes sense in this context. To confirm this finding we would need to perform a control experiment in which we provide both, matching and mismatched information about the type of the emotion. Even though distinctness is not a predictor for convincingness, communication would fail between the machine and the user if the expression is frequently misinterpreted. The expression would convince the user of the wrong circumstances.

The subjects were explicitly instructed to distinguish between trustworthiness and convincingness. However, the strong correlation between them and the high $\mathrm{R}$ Square (0.756) suggests that the concepts of convincingness and trustworthiness are not distinct enough to be evaluated separately. We believe that the subjects might have treated them as synonyms.

In this study, the type of emotion has the strongest influence on convincingness. The two "positive" emotions happiness and surprise are rated highest on almost all variables. Anger and especially fear were rated lowest. Highly abstracted faces were as convincing as natural faces. Only the distinctness of an expression was influenced by its abstraction. Interestingly, the Baldi face (94\%) scored higher than the natural face $(89 \%)$. The quality of synthetic facial expression has reached the level of natural faces. The source of the emotional expression had no influence on its convincingness. This result is in line with the media equation [4].

\section{CONCLUSIONS}

The affective expressions of machines are as convincing as expressions of humans. Our results support the work of Nass and Reeves [4]. We showed that abstracted expressions are as convincing as natural human faces. Their distinctness, however, decreases with a higher level of abstraction. At a certain point, communication would fail due to frequent misinterpretations of the expressions. This problem can be avoided by leaving out less distinct emotion categories, such as fear. Both, the influence of the context and the relation between gradients of intensity and appropriateness are interesting subjects for further research.

In short, the vocabulary of emotional expressions is working, but further research on the grammar and the etiquette is necessary.

\section{REFERENCES}

1. Bartneck, C., Affective Expressions of Machines, Eindhoven, Stan Ackerman Institute, 2000. Also: http://www.bartneck.de/work/aem.pdf

2. CSLU Toolkit.available at http://cslu.cse.ogi.edu/toolkit

3. Fogg, B.J. and Hsiang Tseng, The elements of computer credibility, in Proceedings of CHI '99 (Pittsburgh PA, May 1999), ACM Press, 80-87.

4. Nass, C. and Reeves, B., The Media equation, SLI Publications, Cambridge University Press, Cambridge, 1996 